\title{
La fundamentación intuicionista de la matemática*
}

El objetivo que el matemático intuicionista se propone es el siguiente: practicar matemática como una función natural del intelecto, como una libre, vital actividad del pensamiento. Para él la matemática es un producto de la mente humana. Utiliza el lenguaje, tanto el común como el formalizado, sólo para la comunicación, esto es, para inducir a los demás o a sí mismo la contemplación de sus ideas matemáticas. Tal acompañamiento lingüístico no es una representación de la matemática ni, menos, la matemática misma.

Se satisfará mucho mejor el enfoque activo del intuicionista si pasamos directamente a la construcción de la matemática. Su componente más importante es el concepto de unidad, principio arquitectónico del que depende la serie de los números enteros. Tales números enteros deben ser considerados como unidades que sólo se diferencian unas de otras a través de su lugar en la serie. Aquí me disculpo de continuar con estos conceptos que ya Natorp en su "Fundamentos lógicos de las ciencias exactas" ${ }^{1}$ ha desarrollado de un modo que coincide bastante bien, en lo fundamental, con la manera intuicionista de pensar. Tengo que adelantar sin embargo una observación esencial, a saber, que nosotros no atribuimos a los números enteros, y congruentemente a otros objetos matemáticos, una existencia independiente de nuestro pensamiento, o sea, una existencia trascendente. Tal vez sea cierto que cada idea hace referencia a un determinado objeto, pensado como independiente de ella; podemos dejarlo abierto. De todos modos, estos objetos necesitan no ser totalmente independientes del pensamiento humano. Los objetos matemáticos, aun cuando sean tal vez independientes de un acto individual de pensamiento, son por su esencia condicionados por el pensamiento humano. Su existencia sólo está asegurada en tanto puedan ser determinados por él mismo y tendrán propiedades en tanto éstas puedan ser reconocidas en ellos a través del pensamiento. Pero la posibilidad de este conocimiento sólo se nos manifiesta a través del reconocimiento mismo. La creencia en la existencia trascendente tal que no esté sostenida por los conceptos debe ser rechazada como medio matemático de prueba. Como he de discutir enseguida con un ejemplo, aquí yace el fundamento de la duda sobre la ley del tercero excluido.

Oscar Becker se ha ocupado detalladamente del problema de la existencia matemática en su libro sobre el tema y ha descubierto también algunas conexiones entre estas preguntas y los problemas filosóficos más profundos.

Volvamos ahora a la construcción de la matemática. Con la introducción de las fracciones como pares de números enteros no hay dificultades básicas; distinto es con la definición de los números irracionales. Un número real, tras Dedekind, se define asignando a cada número racional o bien el predicado "izquierda" o bien el predicado "derecha", de modo tal que quede preservado el orden natural de los números racionales. Si quisiéramos admitir esta definición, exactamente así, en la matemática intuicionista, entonces no sería seguro que la constante $\mathrm{C}$ de Euler fuese un número real. No se necesita la definición de c; alcanza con saber que ella supone un algoritmo que permite encerrar a c dentro de un intervalo racional arbitrariamente pequeño. (Un intervalo racional es un intervalo cuyos puntos finales son números racionales; la expresión "encerrar" es inexacta, ya que todavía no se ha definido ninguna relación de orden entre $\mathrm{C}$ y los números racionales, pero práctica y apropiada; se

\footnotetext{
* Traducción de Heyting, A. (1931), “Die intuitionistische Grundlegung der Mathematik”, Erkenntnis 2: 106-115.

1 Natorp, P. (1910), Die logischen Grundlagen der exakten Wissenschaften, Leipzig/Berlin: B. G. Teubner. [N. de la T.] Metatheoria 10(2)(2020): 73-78. ISSN 1853-2322. eISSN 1853-2330.

(C) Editorial de la Universidad Nacional de Tres de Febrero.

(C) Editorial de la Universidad Nacional de Quilmes.

Publicado en la República Argentina.
} 
trata de la computación de una serie de intervalos racionales tales que cada uno está contenido en el anterior y tal que ella pueda continuarse hasta que el último intervalo sea más pequeño que un límite previamente dado de modo arbitrario.) Pero a través de este algoritmo no se ha dado todavía ningún recurso para decidir, para un número racional arbitrario $A$, si se halla a la izquierda o a la derecha de C, o si tal vez es el mismo C, y eso es lo que la definición de Dedekind comprendida intuicionistamente requeriría.

Ahora bien, la crítica usual a este razonamiento es que no importa que esto no pueda decidirse, ya que si $\mathrm{A}=\mathrm{C}$ no fuese el caso, entonces o bien $\mathrm{A}<\mathrm{C}$ o bien $\mathrm{A}>\mathrm{C}$ y esto último se decide tras una finita, aunque quizá desconocida cantidad $N$ de pasos en la computación de C. Sólo necesito formular la crítica de otra manera para refutarla. Ella sólo puede significar lo siguiente: o bien existe un número natural $\mathrm{N}$ con la propiedad de que tras $\mathrm{N}$ pasos en la computación de $\mathrm{C}$ resulta que o bien $\mathrm{A}<\mathrm{C} \mathrm{o}$ bien $\mathrm{A}>\mathrm{C}$, o ese número $\mathrm{n}$ no existe, por lo tanto, obviamente, $\mathrm{A}=\mathrm{C}$. Pero, como hemos visto, la existencia de $\mathrm{N}$ no significa otra cosa que la posibilidad de mostrar efectivamente un número con la propiedad exigida, y la inexistencia significa la posibilidad de derivar una contradicción a partir de dicha propiedad. Como no sabemos si una de estas posibilidades existe, no podemos afirmar, que $n$ exista o no. En este sentido se puede decir que la ley del tercero excluido no debe ser usada aquí.

La definición de Dedekind es, por lo tanto, inutilizable para la matemática intuicionista en su forma original. Brouwer la ha mejorado del siguiente modo: piénsense los números racionales enumerados de alguna manera; por razones de simplicidad me restrinjo a los números del intervalo cerrado de la unidad y tomo la siguiente enumeración como básica:

$$
0,1, \frac{1}{2}, \frac{1}{3}, \frac{2}{3}, \frac{1}{4}, \frac{3}{4}, \frac{1}{5}, \frac{2}{5}, \frac{3}{5}, \frac{4}{5}, \ldots \ldots \ldots
$$

Un número real es determinado por un corte en la serie (A), esto es, a través de una regla que asigna a cada número racional de la serie el predicado "izquierda" o "derecha" de modo que el orden natural de los números racionales se preserva, pero en cada paso se deja un único número fuera de esta correspondencia. Por ejemplo: sea la regla conformada de tal manera que la serie de predicados comienza así:

$$
\begin{aligned}
& \text { O, } 1, \frac{1}{2}, \frac{1}{3}, \frac{2}{3}, \frac{1}{4}, \frac{3}{4}, \frac{1}{5}, \frac{2}{5}, \frac{3}{5}, \frac{4}{5}, \ldots \ldots \ldots \\
& \text { i, d, i, i, ?, i, }
\end{aligned}
$$

Aquí $\frac{2}{3}$ se ha dejado temporariamente fuera de la correspondencia; no es necesario saber si el predicado para $\frac{2}{3}$ se determinará en algún momento, pero es también posible que $\frac{3}{4}$ aparezca como el nuevo número excluido y, entonces, $\frac{2}{3}$ reciba el predicado "izquierda".

Es sencillo ofrecer un corte para la constante de Euler. Sea $d_{n}$ la diferencia más pequeña entre dos números sucesivos entre los primeros $n$ números de (A); si ahora se computa $c$ hasta llegar a un intervalo racional $i$ menor a $d_{n}$, entonces podrá a lo sumo uno de estos $n$ números caer dentro de $i$; si existe tal número, será el número excluido para el corte. Podrá observarse cuán estrechamente vinculada está la definición de Brouwer con la efectiva computación de un número real.

Ahora se puede dar un importante paso adelante y abandonarse la exigencia de que la serie predicativa esté determinada hasta el infinito a través de una regla; alcanza con que de alguna manera, por ejemplo a través de elecciones libres, ella se vaya determinando paso a paso; llamo a tales sucesiones indefinidamente continuables. La definición de número real se amplía, por lo tanto, de manera que además de sucesiones conforme a regla permita también sucesiones indefinidamente continuables. Antes de estudiar más en detalle esta nueva definición, veremos un ejemplo sencillo. Comienzo con una sucesión de elección izquierda-derecha:

$$
\begin{aligned}
& \text { O, } 1, \frac{1}{2}, \frac{1}{3}, \frac{2}{3}, \frac{1}{4}, \frac{3}{4}, \frac{1}{5}, \frac{2}{5}, \frac{3}{5}, \frac{4}{5}, \ldots \ldots \ldots \\
& \text { i, d, i, i, d, i, d, i, i }
\end{aligned}
$$


Aquí la pregunta de qué predicado recibe $\frac{3}{5}$ no puede responderse todavía, ya que eso todavía debe ser decidido; mientras que la pregunta por el predicado de $\frac{4}{5}$ puede responderse con "derecha” ya que es lo que vale para cada continuación de la serie. En general, son sólo aquellas preguntas relativas a una secuencia indelimitadamente continuable las capaces de recibir una respuesta determinada, vinculada con cada continuación posible de la secuencia; las otras preguntas, como la de arriba acerca del predicado $\frac{3}{5}$, deben ser consideradas sin sentido. La secuencia electiva reemplaza, así, no tanto a las secuencias conforme a regla individuales sino más bien a la totalidad de las reglas posibles. Una secuencia electiva derecha-izquierda, cuya libertad sólo está restringida por las condiciones preestablecidas por el orden natural de los números racionales, no determina un número real sino el conjunto de todos los números reales, o sea, el continuo. Mientras que de la otra manera cada número real se piensa individualmente definido y luego se los reúne, aquí el continuo se lo define como un todo. Cuando se restringe la libertad de elección a través de una regla previamente dada se obtienen conjuntos de números reales; por ejemplo, si yo prescribo que la secuencia debe empezar tal como se ha escrito, defino el conjunto de los números reales entre $\frac{1}{2} \mathrm{y} \frac{2}{3}$. A través de un acotamiento cada vez mayor de la libertad de elección, una secuencia indefinidamente continuable se va convirtiendo en una secuencia conforme a regla.

He usado la palabra "conjunto" [Menge] exactamente en el sentido brouweriano; su definición de conjunto es una generalización de esta formación de conceptos. Además de las secuencias de elección, Brouwer considera también aquellas secuencias que se constituyen a través de una regla de correspondencia a partir de una secuencia de elección. Un conjunto tiene dos reglas: la primera establece qué elecciones de números naturales son permitidas luego de que se haya efectuado una determinada serie finita de elecciones permitidas. Esto debe hacerse de modo tal que como mínimo una nueva elección permitida pueda conocerse luego de cada serie finita de elecciones permitidas. El orden natural de los números racionales es un ejemplo de regla de este tipo para la secuencia izquierdaderecha considerada arriba. La segunda regla establece que cada elección permitida asigna un objeto matemático que puede depender, obviamente, de las elecciones ya hechas; así, queda permitido que la asignación finalice con una elección determinada y, por lo tanto, que cada siguiente elección no asigne más nada. Una sucesión que resulta de una sucesión de elección permitida a través de la regla de asignación se denomina elemento del conjunto.

Para llevar el ejemplo previamente desarrollado del conjunto de números racionales entre $\frac{1}{2}$ y $\frac{2}{3}$ a esta definición general, reemplacemos los predicados "derecha", "izquierda" y "temporariamente indeterminado" por 1, 2 y 3 , obtengamos la regla para las elecciones permitidas del orden natural de los números racionales y del requerimiento de que la secuencia comience de un modo determinado y elijamos como regla de asignación la identidad.

Un conjunto no es la sumatoria de sus elementos (esto no tiene sentido, si no se lo considera como existente en sí mismo) sino que se identifica con la regla que lo define. De dos elementos de un conjunto se dice que son iguales, si, en ambos, para cada $n$ iguales objetos están en el $n$-ésimo lugar. La igualdad de elementos de un conjunto no significa por tanto que ellos sean el mismo elemento; para ello, deberían ser asignados a la misma secuencia electiva en el mismo conjunto. Sería impráctico denominar iguales a dos objetos matemáticos solamente cuando son el mismo objeto; más bien debe darse para cada tipo de objetos una propia definición de igualdad.

Brouwer llama especie a los conjuntos que están definidos, según la terminología clásica, a través de una propiedad característica de sus elementos. Una especie, tanto menos que un conjunto, no es la suma de sus elementos sino que se identifica con su propiedad definitoria. Así, las definiciones impredicativas son imposibles, lo que es evidente para los intuicionistas, ya que como elementos de una especie sólo pueden figurar objetos previamente definidos. De acá resulta una introducción gradual de las especies. El primer nivel lo forman las especies conjuntistas [Mengenspezies] cuya propiedad definitoria consiste en la identidad con un elemento de un conjunto determinado; así, a cada conjunto $\mathrm{M}$ pertenece la especie conjuntista cuyos elementos conjuntistas [Mengenelemente] son 
iguales a un elemento de $\mathrm{M}^{1}$ ) Una especie de primer orden puede contener elementos conjuntistas y especies conjuntistas; una especie de segundo orden tiene además especies de primer orden como elementos, etc.

La introducción de las sucesiones indefinidamente continuables no se sigue con necesidad del punto de vista intuicionista. Se podría construir una matemática intuicionista sin sucesiones de elección. Con el siguiente teorema de la teoría de conjuntos sobre el continuo, cuya demostración puede servir asimismo como ejemplo de un razonamiento intuicionista, mostraré cuánto se empobrecería de este modo la matemática.

Sea asignado a cada número real un número natural a través de una ley. Asumimos que los números reales $a$ y $b$ tienen números asignados diferentes, por ejemplo, 1 y 2 . Entonces se puede determinar a través de una construcción sencilla un tercer número $c$ que tenga la siguiente propiedad: dada cualquier proximidad de $c$, no importa cuán pequeña, existe un número distinto de $c$ que es un número asignado [genummerte Zahl]; o sea, cada segmento inicial finito del corte que define c puede continuarse de modo tal de obtener un número asignado [genummerte Zahl] que sea distinto de c. Ahora defino el número $d$ a través de una secuencia de elección como sigue: comienzo como con $c$ pero me reservo la libertad, frente a una elección arbitraria, de continuar de manera diferente de como se hizo con c. Obviamente el número de $d$ no será determinado por ninguna cantidad finita previamente conocida de elecciones; de lo que se sigue que $d$ no tiene asignado ningún número determinado. Eso contradice la premisa de que cada número real tiene un correlato natural [Nummer], de modo que nuestra asunción de dos números asignados y diferentes $a$ y $b$ lleva a contradicción. Siendo que dos números naturales que no pueden distinguirse son iguales, tenemos el siguiente teorema:

$\mathrm{Si}$ a cada número real se le asigna un número natural, entonces todos tienen el mismo número.

Como caso particular se tiene:

Si un continuo se parte en dos subespecies de modo que cada elemento pertenezca a una y sólo una de estas subespecies, entonces una subespecie es vacía y la otra idéntica al continuo.

La unidad del continuo no puede, por ejemplo, partirse en la especie de los números entre 0 y $\frac{1}{2}$ y en la especie de los números entre $\frac{1}{2}$ y 1 ; la construcción precedente arroja aquí un número del que nunca se necesitará decidir si es menor o mayor que $\frac{1}{2}$. Los teoremas sobre la continuidad de una determinada función en un intervalo también están conectados con el teorema anterior; el teorema de Brouwer sobre la continuidad uniforme de toda función completa, en cambio, sobrepasa en mucho estos resultados.

Nos preguntamos ahora qué resulta del teorema probado arriba si no se permite en la matemática ninguna sucesión indefinidamente continuable. En el lugar del continuo debería estar entonces la especie de los números definidos a través de sucesiones conforme a leyes. Esta definición es admisible sólo si se la comprende como afirmando que un número pertenece a esta serie cuando hay una regla que permite efectivamente determinar todos los predicados de la sucesión uno tras otro.

La demostración anterior sigue siendo válida para este caso sólo si se logra definir el número $d$ a través de una sucesión conforme a regla en lugar de hacerlo a través de una sucesión electiva. Y eso es fácilmente asequible si podemos hacer uso de algún problema irresuelto, por ejemplo de la pregunta sobre la aparición de la secuencia 0123456789 en el desarrollo decimal de $\pi$; se puede hacer depender la cuestión de la posibilidad del desvío de la sucesión de predicados para c en el predicado n-ésimo de la secuencia de predicados para $d$ de la aparición de una secuencia en la n-ésima cifra luego del punto decimal de $\pi$. Esta demostración obviamente caerá tan pronto como la cuestión sobre tal secuencia sea solucionada; pero entonces se la podrá reemplazar por otra irresuelta similar, si todavía persiste alguna. El teorema sobre las sucesiones conforme a regla sólo puede demostrarse bajo la condición de que siempre existan problemas irresueltos. Más precisamente: el teorema es correcto si hay dos números determinados a través de tales sucesiones para los que la pregunta de si son iguales o diferentes

1) La definición de especie conjuntista la tomo de una comunicación del Prof. Brouwer. 
constituye un problema que se demuestra irresoluble; es falso, si la existencia de dos números tales resulta absurda. Pero el problema que surge a partir de estas condiciones es inabordable; incluso aquí las sucesiones electivas se muestran superiores a la sucesiones conforme a regla, dado que independizan a la matemática de la pregunta sobre la existencia de problemas irresolubles.

Concluyo aquí mis observaciones sobre la construcción de la matemática para agregar algo sobre la lógica proposicional intuicionista. Aquí se distingue entre proposiciones y aserciones: una aserción es la aseveración de una proposición. Una proposición matemática expresa una determinada expectativa; por ejemplo la proposición "La constante $\mathrm{C}$ de Euler es racional" significa la expectativa de que se pueden encontrar dos números enteros $a$ y $b$ tal que $C=a / b$. Quizá la palabra "intención”, acuñada por los fenomenólogos, expresa aún mejor lo que acá se quiere decir que la palabra "expectativa”. Uso también la palabra "proposición" para la intención que es expresada lingüísticamente por la proposición. La intención, como ya se enfatizó anteriormente, se dirige no a un estado de cosas pensado como existente independientemente de nosotros sino a un acontecimiento pensado como posible, como lo muestra claramente el ejemplo de arriba.

La aseveración de una proposición significa la satisfacción de la intención, por ejemplo, la aseveración "C es racional" significaría que se han encontrado de hecho los números enteros requeridos. Yo distingo la aseveración de la correspondiente proposición mediante el signo de aseveración $\vdash$ que procede de Frege y fue usado también por Russell y Whitehead con este objetivo. La aseveración de una proposición no es a su vez una proposición sino la constatación de un hecho empírico, a saber, la satisfacción de la intención expresada mediante la proposición.

Una función lógica es un procedimiento para construir otra proposición a partir de una proposición dada. La negación es una función tal; en continuidad con Husserl, Becker ha descripto su significado con mucha precisión. Según él, la negación es algo completamente positivo, a saber, la intención de un conflicto ligado con la intención originaria. $\mathrm{O}$ sea, la proposición "C no es racional" significa la expectativa de derivar una contradicción de la suposición de que c sea racional. Es importante notar que la negación de una proposición hace referencia siempre a un procedimiento de prueba que conduce a una contradicción incluso cuando la proposición original no haga mención a procedimiento alguno. Como signo para la negación uso $\neg$.

Para la ley del tercero excluido utilizamos la función lógica "o bien... o bien". $p \vee q$ significa la intención que es satisfecha si y sólo si al menos una de las intenciones $p$ o $q$ es satisfecha. La fórmula para la ley del tercero excluido sería $\vdash p \vee \neg p$. Esta ley recién puede entonces afirmarse para una determinada proposición $p$ si $p$ o bien puede probarse o bien probarse de ella una contradicción. Una demostración para la ley general debería consistir en la propuesta de un método por el cual, dada una proposición cualquiera siempre pueda probarse esa proposición o su negación. La fórmula $p \vee \neg p$ significa entonces la expectativa de una construcción matemática (un método de prueba) que satisfaga esta exigencia; o sea, la fórmula es una proposición matemática, y la pregunta acerca de su validez es una pregunta matemática que, en el mejor de los casos, es resoluble por medios matemáticos. En este sentido la lógica es dependiente de la matemática.

Concluyo con algunas observaciones sobre la pregunta acerca de la solubilidad de los problemas matemáticos. Un problema es dado a través de una intención que se busca satisfacer. Es solucionado cuando o bien la intención se satisface con una construcción o se demuestra que ella conduce a una contradicción. La pregunta sobre la solubilidad puede, por lo tanto, retrotraerse a la de la demostrabilidad.

Una demostración de una proposición es una construcción matemática que puede ella misma ser tratada matemáticamente. La intención de tal prueba conduce, por tanto, a una nueva proposición; si simbolizamos la proposición: "la proposición p es demostrable" con $+p$, entonces + es una función lógica, la "demostrabilidad". Las aseveraciones $\vdash p$ y $+p$ tienen exactamente el mismo significado; ya que si $p$ está demostrada, entonces también está demostrada la demostrabilidad de $p$, y si + $p$ está demostrada, entonces la intención de una demostración para $p$ se satisface, esto es, se ha demostrado $p$. Sin embargo las proposiciones $p \mathrm{y}+p$ no son idénticas, como se mostrará claramente con un ejemplo. 
En la computación de la constante $\mathrm{C}$ de Euler puede ocurrir que un valor racional determinado, digamos $\mathrm{A}$, se mantenga por un tiempo inusualmente largo contenido en el intervalo en el que vamos acotando $C$ más y más de manera que finalmente sospechamos que $C=A$, o sea que esperamos que continuando nuestra computación, seguiremos encontrando siempre a A dentro de nuestro intervalo. Pero esta suposición no es una prueba de que ello vaya a pasar siempre. Por tanto, la proposición $+(\mathrm{C}$ = A) contiene más que $(C=A)$.

Si se aplica la negación sobre estas dos proposiciones, entonces se obtienen no sólo dos proposiciones diferentes $\neg p$ y $\neg^{+} p$, sino que también las aseveraciones $\vdash \neg p$ y $\neg \neg^{+} p$ son diferentes. $\vdash$ $\neg^{+} p$ significa que la asunción de una construcción como $+p$ exige es contradictoria; por lo tanto, la simple expectativa $p$ no necesita todavía conducir a una contradicción. En nuestro ejemplo esto es así: supongamos que la asunción de una construcción que prueba que $A$ está en cada intervalo que contiene a $\mathrm{C}$ resulta contradictoria $(\vdash \neg+p)$; entonces no necesariamente la asunción de que en la computación efectiva de $\mathrm{C}$ de hecho siempre encontraremos a $\mathrm{A}$ en nuestro intervalo llevará a contradicción. Incluso es pensable el caso de que podríamos probar que la última asunción podría no probarse nunca como contradictoria de modo que pudiésemos aseverar $\vdash \neg^{+} p$ y $\vdash \neg \neg p$ al mismo tiempo. En tal caso la pregunta de si $c=a$ sería esencialmente insoluble.

La diferencia entre $p$ y $+p$ desaparece ni bien en $p$ misma se intenta una construcción, ya que la posibilidad de una construcción sólo puede ser demostrada a través de su realización efectiva. Cuando uno se restringe a tales proposiciones, en las que se exige una construcción, no surge la función lógica de la demostrabilidad en absoluto. Esta restricción puede ser alcanzada si se consideran solamente proposiciones de la forma " $p$ es demostrable" o, dicho de otra manera, si se piensa, para la satisfacción de cada intención, la intención añadida de una construcción. La lógica intuicionista debe ser comprendida en este sentido, tal como ha sido desarrollada hasta aquí, sin la utilización de la función +. La introducción de la demostrabilidad conllevaría grandes complicaciones; y, dado su reducido valor práctico, no vale la pena lidiar con ellas en detalle. $\left.{ }^{1}\right)$ Aquí el concepto nos ha permitido ver cómo concebir un problema esencialmente insoluble.

Habré alcanzado mi propósito si pude mostrar que el intuicionismo no contiene asunciones arbitrarias y, aún menos, prohibiciones artificiales tales que permitan evitar las paradojas lógicas, sino que él elabora el único camino posible para, una vez asumida una posición básica, construir la matemática.

Arend Heyting

Traducción: María Gabriela Fulugonio ${ }^{\dagger \ddagger}$

\footnotetext{
$\left.{ }^{1}\right)$ La cuestión tratada en este párrafo recién fue clarificada completamente en una discusión con H. Freudenthal luego de las Jornadas; el resultado de esta discusión está reproducido en el presente texto.

+ Universidad de Buenos Aires, Argentina. Para contactar a la autora, por favor, escribir a: gabrielafulugonio@gmail.com.

* Agradezco a Javier Legris y a Pablo Lorenzano los comentarios y sugerencias realizados a la presente traducción. [N. de la T.]
} 